\title{
Krise der Raumplanung - aus der Sicht der Praxis IN ÖSTERREICH
}

\author{
Friedrich SCHINDEGGER, Wien*
}

mit 2 Abb. im Text

\section{INHALT}

Summary 159

Zusammenfassung.....

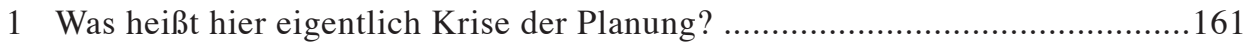

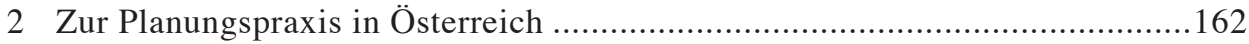

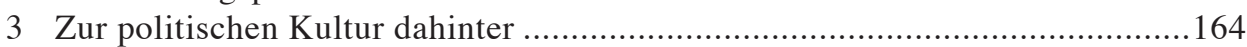

4 Konkrete Erfahrung: Siedlungspolitik ignoriert Forschungsergebnisse ...........165

5 Zur Neuorientierung am Gemeinwohl ...................................................... 166

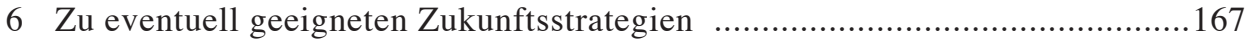

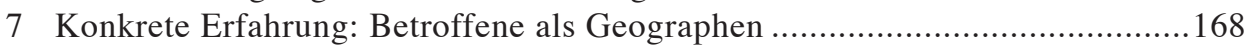

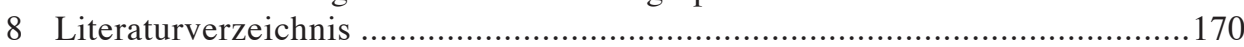

\section{Summary}

Crisis of spatial planning - An Austrian practical experience.

The contribution shows a lecture held at the Congress of German Geographers in Vienna 2009. A wide-spread perception of spatial planning is based on misunderstandings concerning the terms space and planning. The development of planning in Austria is problematic, however not precarious in the sense of a recognisable height or turning point, but rather characterised by permanent system-inherent weaknesses. Background for that is a political culture, in which planning is not regarded as a political instrument, rather than as a restriction of the political (spontaneous) scope of action. The confusing distribution of competences is recognised not as problem, but forms the arena for contests between different planning authorities.

* Dipl.-Ing. Dr. Friedrich SChINDEGGER war langjähriger Mitarbeiter des Österreichischen Instituts für Raumplanung (ÖIR); e-mail: friedrich.schindegger@gmx.at 
Public welfare is not in the focus of the political main stream. It is, however, actually the substantial socio-political legitimation of spatial planning at all. Right by means of spatial problems it can be made relatively easily comprehensible, what is public welfare about. Spatial planning is subject to political calculation. Thus, for achieving a wider acceptance of spatial planning in the public it needs a strategic reorientation. The central task is striving for a majority at the opinion market. Therefore, a basic condition is a perception of space, which recognises the spatial relations of real life and introduces space as a dimension of responsibility into the political discourse. The example of the planning process project 'vision rheintal'(Vorarlberg) shows, how citizens and actors involved become themselves geographers and thus develop a learning society.

\section{Zusammenfassung}

Der Beitrag gibt einen Vortrag am Deutschen Geographentag in Wien 2009 wieder. ${ }^{1)}$ Einem weit verbreiteten Aufgabenverständnis von Raumplanung liegen Missverständnisse bezüglich Raum und Planung zugrunde. Die Entwicklung in Österreich ist zwar problematisch, jedoch nicht krisenhaft im Sinne eines erkennbaren Höhe- oder Wendepunktes, sondern vielmehr von permanenten systemimmanenten Schwächen gekennzeichnet. Hintergrund ist eine politische Kultur, in der Planung nicht als politisches Instrument begriffen wird, sondern als Beschränkung des politischen (spontanen) Handlungsspielraums. Die verwirrende Kompetenzlage wird nicht als Problem erkannt, sondern bildet die Arena für Auseinandersetzungen zwischen verschiedenen Planungsträgern.

Gemeinwohl ist nicht im Visier des politischen "main streams". Es ist aber eigentlich die wesentliche gesellschaftspolitische Legitimation der Raumplanung überhaupt. Dabei könnte gerade an den Problemen im Raum relativ leicht verständlich gemacht werden, worum es geht. Raumplanung ist dem politischen Kalkül unterworfen. Um eine größere Akzeptanz der Raumplanung in der Öffentlichkeit zu erzielen, bedarf es einer strategischen Umorientierung. Zentrale Aufgabe wäre das Gewinnen einer Mehrheit am Meinungsmarkt. Grundvoraussetzung für die Mehrheitsfähigkeit ist eine Raumwahrnehmung, welche die lebensweltlichen Zusammenhänge im Raum erkennt und dementsprechend den Raum als Verantwortungsdimension in den politischen Diskurs einführt. Der Beispielfall des Vorarlberger Planungsprozessprojekts, vision rheintal'zeigt, wie die Betroffenen und Akteure selbst zu Geographen und zu einer lernenden Gesellschaft werden.

\footnotetext{
1) Der Vortragsstil wurde auch in dieser schriftlichen Ausführung bewusst beibehalten.
} 


\section{Was heißt hier eigentlich Krise der Planung?}

Wir reden hier von Raumplanung. Und damit befinden wir uns mitten in einem Minenfeld von Missverständnissen um die Begriffsinhalte von Raum und Planung. Diese Minen werden hinterlassen von den verschiedenen Disziplinen, die dieses Feld beackern. So läuft die im Allgemeinen theorieschwache, um nicht zu sagen theoriefeindliche Profession Raumplanung schon bei der Klärung ihres Selbstverständnisses in zahlreiche Planungsfallen. Raumplaner kümmern sich zumeist weder um die verschiedenen Raumkonzepte der Geographie, noch um die Diskussion von Modellen der Planungstheorie; und ebenso wenig um die Tatsache, dass Raumplanung ein wesentlicher Teil und sehr greifbarer Ausfluss der politischen Kultur eines Landes ist (in der Sprachregelung der Europäischen Union heißt das heute Territorial Governance).

Wovon reden wir also, wenn wir fragen, ob die Planung in der Krise ist? Da die Frage nach der Perspektive der Planungspraxis in Österreich gestellt worden ist, wird auf den theoretischen Zugang verzichtet. Obwohl man sich natürlich schon fragen kann, ob mangelnde empirische Fundierung, logische Inkonsistenz und unreflektierte Begriffssysteme nicht schon von vornherein die Entwicklung einer erfolgreichen Planungspraxis in Frage stellen.

Von der Planungspraxis in Österreich redend, bleibt hier also nichts anderes übrig, als die Frage nach der Krise der Raumplanung vor dem Hintergrund jenes Selbstverständnisses zu behandeln, das den Gesetzen, Programmen, Plänen und Konzepten zu entnehmen ist, ohne dieses weiter zu hinterfragen. Und dennoch kommt es auch hier innerhalb dieser pragmatischen Perspektive zu Missverständnissen. Auf die so plausible Frage: „Wer plant den Raum?“ gibt es keine Antwort, denn die Frage ist zweifach falsch gestellt. Erstens ist nicht der Raum der Planungsgegenstand. Zweitens knüpft sie an ein Planungsverständnis an, das aus dem Bauwesen kommt. Dort

- ist der Gegenstand der Planung ein physisches Objekt;

- bestimmende Faktoren sind die Auftraggeberbedürfnisse von Individuen oder institutionellen Bauträgern;

- die Planrealisierung besteht in der direkten bautechnischen Umsetzung des Planes;

- der Verzicht auf Planung bedeutet Verzicht auf das Objekt, womit es keine Veränderung gibt.

Demgegenüber betrifft Raumplanung

- die weitere Entwicklung räumlicher Strukturen - d.h. physische und/oder sozioökonomische Strukturen und funktionelle Beziehungen im Raum;

- Auftraggeber ist die Gesellschaft, vertreten durch öffentliche Gebietskörperschaften der verschiedenen Ebenen;

- bestimmende Faktoren sind Raumplanungsziele und -konzepte, das verfügbare Instrumentarium sowie die politische Willensbildung;

- die Planrealisierung erfolgt durch die indirekte Einflussnahme auf öffentliche und private Entscheidungen bezüglich dieser räumlichen Strukturen - Raumplanung verfügt über keine eigene Umsetzungsmacht (mit Ausnahme der meist nachgeordneten Baubehörden); 
- Raumplanung kann also in aller Regel nur Einfluss nehmen, wenn gebaut wird, nicht aber dass gebaut wird;

- der Verzicht auf Planung bedeutet im Falle der Raumplanung, dass diese räumlichen Strukturen in jedem Fall Veränderungen unterworfen werden und sich weiter entwickeln, jedoch ohne dass auf die Ziele der Raumplanung Rücksicht genommen wird.

Raumplanung ist also jener permanente Prozess, der die Grundlagen dafür schafft, dass die öffentliche Hand - entsprechend gesetzlich geregelten Zielsetzungen und Verfahrensvorschriften - auf die Entwicklung der räumlichen Verhältnisse Einfluss nehmen kann. Raumplanung unterscheidet sich damit von Bauplanung wesentlich durch Träger, Gegenstand, Ziele und Mittel. Das ist jedoch den meisten, die nicht aus beruflichen Gründen direkt mit Raumplanung zu tun haben, nicht geläufig. Somit tragen darauf gründende falsche Erwartungen oder auch Befürchtungen schon immer wesentlich zu einer „Krise der Raumplanung“ bei.

Noch ein Wort zur Krise. Der Duden definiert Krise als eine „schwierige Situation oder Zeit, die den Höhe- und Wendepunkt einer gefährlichen Entwicklung darstellt““. Aus den folgenden Ausführungen lässt sich jedoch ableiten, dass die Entwicklung in Österreich zwar als problematisch erscheint, jedoch weder ein Höhe- noch ein Wendepunkt, sondern eher permanente systemimmanente Schwächen zu erkennen sind. Typisch für die Situation - vielleicht nicht nur in Österreich - ist jedenfalls, dass in offiziellen Berichten keine Spur von Krise oder krisenhafter Selbsteinschätzung zu finden ist. Im privaten Gespräch mit Vertretern der planenden Verwaltung trifft man jedoch sehr wohl auf realistische Einschätzungen der Systemschwächen. Die Konsequenz aus diesem Widerspruch äußert sich allerdings vielfach in Resignation und fallweise auch Zynismus.

Von den Systemschwächen und schließlich von eventuell geeigneten Gegenstrategien soll im Folgenden die Rede sein.

\section{Zur Planungspraxis in Österreich}

Zunächst ist etwas vorauszuschicken, das im öffentlichen Bewusstsein nicht so differenziert wahrgenommen wird - wenn überhaupt eine einigermaßen zutreffende Vorstellung von Raumplanung gegeben ist: Raumplanung vollzieht sich auf verschiedenen Ebenen, nicht nur deshalb, weil sie einer bestimmten Kompetenzverteilung folgt, sondern grundsätzlich schon deshalb, weil die Wirklichkeit der gesellschaftlichen Lebensbezüge sich - und zwar gleichzeitig - in lokalen, regionalen, nationalen, europäischen und globalen Zusammenhängen vollzieht. Dass sich reale Funktionsräume immer stärker über territoriale Grenzen von Gebietskörperschaften hinweg entwickeln, wirft dabei noch ganz andere Fragen auf, die aber hier nicht behandelt werden können.

Die Praxis der österreichischen Raumplanung und das damit verbundene Aufgabenverständnis ist weitgehend von der Flächenwidmung auf der lokalen Ebene, das heißt, durch die Gemeinden beherrscht. Das ist gleichzeitig die einzige Ebene, auf der 
Raumplanung flächendeckend stattfindet. Flächenwidmungspläne liefern in der politischen Realität vor allem die Legitimation der jeweils aktuellen durchsetzungsfähigen Nutzungsansprüche. So sind Standorte und Strukturen vielmehr die Folge von individuellen ad hoc-Übereinkünften von Investoren, Liegenschaftseigentümern und Akteuren des politischen Systems, als das Ergebnis vorausschauender staatlicher Lenkung.

Die Ebene der Länder, in Österreich etwa so groß wie deutsche Regierungsbezirke, ist von neun verschiedenen Raumordnungsgesetzen geprägt, denen - mangels einer entsprechenden Kompetenz in der Bundesverfassung - kein gemeinsamer bundesgesetzlicher Rahmen zugrunde liegt. Neun Raumordnungsgesetze mit unterschiedlichen Widmungskategorien (kein Bundesbaugesetz wie in Deutschland!) führen zu neun verschiedenen Kombinationen mit Bundesrecht (bezüglich Verkehrstrassenfestlegung, Gewerbe-, Wasser-, Forstrecht usw.), im Übrigen auch mit neun verschiedenen (Landes-)Naturschutzgesetzen. Dies kann eigentlich nicht durch unterschiedliche regionale Erfordernisse gerechtfertigt werden.

So weisen die überörtlichen Raumplanungen der Länder - den je eigenen Raumordnungsgesetzen folgend - erhebliche Unterschiede auf. Insgesamt dürfte ihnen aber - von den nun zunehmend ausgewiesenen Siedlungsgrenzen abgesehen - wenig regulierende Wirkung zukommen. Dazu kommt, dass die verantwortlichen Landesregierungsmitglieder es als Teil ihrer politischen Befugnis betrachten, darüber zu bestimmen, ob und wo die im jeweiligen Gesetz vorgesehenen überörtlichen Planungsinstrumente überhaupt zur Anwendung kommen. Fachexpertise und das Engagement der zuständigen beamteten Planer können sich also - um es nobel zu formulieren - nur innerhalb des jeweils aktuellen politischen Kalküls entfalten. Das gilt übrigens auch für Raumforschungsarbeiten.

Die nationale Ebene ist durch die fehlende Raumplanungskompetenz gekennzeichnet - es gibt auch keine Rahmenkompetenz - um nicht zu sagen davon gezeichnet. Es gibt auch keine gesetzliche Verpflichtung zur Fachplanung jenseits der unmittelbaren Projektplanungsebene (etwa bezüglich der Verkehrsinfrastruktur). Im Rahmen der Österreichischen Raumordnungskonferenz (ÖROK), der zuständigen gemeinsamen Plattform aller Gebietskörperschaften, kann die unkoordinierte Praxis der Raumentwicklungspolitik bestenfalls vergleichend dargestellt, aber nicht harmonisiert werden. Die ÖROK kann auch die Lücke der fehlenden Verantwortung auf der gesamtstaatlichen Ebene nicht füllen. Sie ist eine auf Konsens angewiesene Koordinationsplattform, von der keine politischen Innovationen ausgehen können. Als das wichtigste Produkt dieser Ebene gilt das im zehnjährigen Abstand aktualisierte Österreichische Raumentwicklungskonzept der ÖROK. Dieses hat allerdings meines Erachtens eher die Funktion einer konsensfähigen Zusammenfassung des jeweils aktuellen raumentwicklungspolitischen Bewusstseinsstandes, als den eines richtungweisenden Programms für den praktischen Vollzug von Raumentwicklungspolitik. Es vermeidet - fast bin ich geneigt, zu sagen: grundsätzlich - jeglichen konkreten Raumbezug und entzieht sich mit seinen vagen Zielformulierungen weitgehend der Evaluierbarkeit. Monitoring und Erfolgskontrolle fehlen in der österreichischen Raumplanung völlig.

Schließlich ist in diesem Zusammenhang noch darauf zu verweisen, dass mit der wachsenden Integration Österreichs in die Europäische Union und deren zunehmendem raumentwicklungspolitischem Engagement sich folgende Lücke im österreichischen 
Raumordnungsrecht als fatal erweist: für die Wahrnehmung österreichischer Interessen im Rahmen der europäischen Raumentwicklungspolitik der Europäischen Union verfügt die Republik auf gesamtstaatlicher Ebene über keine Zuständigkeit und damit auch über keine politische Verantwortung für den „territorialen Zusammenhalt“, der im Vertrag von Lissabon zu einer „,von der Union mit den Mitgliedstaaten geteilten Zuständigkeit" erklärt wird.

Diesen unzulänglichen Rechtsgrundlagen der Raumentwicklungspolitik wird nach wie vor mit legislativer Tatenlosigkeit begegnet: Raumordnung war kein Thema des Verfassungskonvents in der vorletzten Legislaturperiode und keines der sogenannten Staats- und Verwaltungsreform in der letzten. Im Programm der derzeitigen Bundesregierung finden sich zwar einige bemerkenswerte Aussagen zur Staatsaufgabe Raumordnung. Sie münden in der Feststellung, dass es „Verhandlungen mit den Ländern über eine Reform der Raumordnung, mit dem Ziel einer Rahmenkompetenz des Bundes (Korridorplanung)“ bedarf. Es gibt jedoch keinerlei Anzeichen dafür, dass diesbezügliche Taten folgen werden - im Schatten der „großen Krise“ schon gar nicht.

\section{Zur politischen Kultur dahinter}

Eine solche Analyse des österreichischen Systems Raumplanung gibt natürlich Anlass, auch auf die politische Umwelt Bezug zu nehmen. Wahrscheinlich ist es nicht übertrieben zu sagen, dass die Raumplanung als Instrument der Gesellschaftspolitik nach einer euphorischen Phase der Selbst- und Fremdüberschätzung in den 1960er und 1970er Jahren seither in der Realpolitik sukzessive an Bedeutung verloren hat. Weder konnten die in den 1980er Jahren aufkommende Umweltorientierung, noch die damals erfolgreich entwickelte Regionalpolitik als „Trägerraketen“ genutzt werden. Im Gegenteil, die rasante Entwicklung des durch europäisches Recht erzwungenen umweltplanerischen Instrumentariums einerseits und die gigantische FördermittelVerteilungsmaschinerie andererseits erwiesen sich eher als Konkurrenten auf dem Markt der politischen Aufmerksamkeit.

An dieser Stelle ist anzumerken, dass im Unterschied zur Raumplanung die verwandten Disziplinen Städtebau und Architektur gerade in den letzten Jahren mehr politische Aufmerksamkeit erringen konnten. Das dürfte daran liegen, dass sie sich besser für politisches und kommerzielles Branding eignen. Insbesondere die überörtliche Dimension der Raumplanung mit ihren ungenutzten Potenzialen und Standortkonkurrenzen lässt sich jedoch schwer bildlich fassen.

Hinderlich für eine Selbstertüchtigung der Raumplanung war und ist meines Erachtens vor allem eine politische Kultur in Österreich, die ganz allgemein von einer ausgesprochenen Planungsphobie gekennzeichnet ist. Planung wird demnach nicht als politisches Instrument begriffen, sondern als Beschränkung des politischen (spontanen) Handlungsspielraumes, als Hindernis für den traditionell praktizierten ad-hocReaktionismus. Die politische Mode der Deregulierung lieferte dafür auch noch die ideologische Rechtfertigung. 
In diesem Milieu vermisst auch niemand das völlige Fehlen auftragsunabhängiger politikorientierter Raumforschungseinrichtungen. Think-tanks wie für die Wirtschaftsund Sozialpolitik gibt es nicht. Bezüglich der Raumentwicklung beansprucht die Politik weitgehend die alleinige Deutungshoheit über Ziele. Im Unterschied zu anderen Politikbereichen wird kein substanzieller Bedarf an wissenschaftlicher Expertise angemeldet.

\section{Konkrete Erfahrung: Siedlungspolitik ignoriert Forschungsergebnisse}

Wie wenig sich Politik an Ergebnissen der Raumforschung orientiert, wenn sie dann doch einmal vorliegen, sei an einem Beispiel erläutert. In der Siedlungsentwicklung versickern jährlich mehrere hundert Millionen Euro an öffentlichen Mitteln, und zwar durch die von den zuständigen Behörden nicht nur zugelassenen, sondern auch noch geförderten Formen der Zersiedlung. Eine vor zehn Jahren von der ÖROK durchgeführte empirisch fundierte Studie ergab, dass mit einer realistisch angesetzten flächen- und infrastrukturkostensparenden Siedlungspolitik wie sie die Raumordnungsgesetze landauf landab vorsehen, in Österreich in zehn Jahren an die 3 Mrd. Euro oder $15 \%$ des Gesamtinvestitionsbedarfs eingespart werden könnten. Das ist etwa darauf zurückzuführen, dass die Erschließungskosten für eine Bebauung mit freistehenden Einfamilienhäusern doppelt so hoch sind wie im verdichteten Flachbau. Noch gravierender sind die Unterschiede bei den Transportkosten für soziale Dienste wie Schülertransport, Heimhilfe und Essen auf Rädern u.ä. Sie steigen bei starker Zersiedlung auf das Zehnfache und darüber hinaus.

Diese Ergebnisse haben bisher so gut wie keine Konsequenzen gezeigt. Die Wohnbauförderung, mit der zwei Drittel des gesamtem Wohnbauvolumens finanziert werden, bietet noch immer zu geringe Anreize für flächensparende Wohnformen und überhaupt keine für raumplanungsgerechte Standortlösungen. Flächensparende Bauweisen finden sich bisher meist nur in Gemeinden mit bereits extremer Flächenknappheit oder sehr hohen Baulandkosten. Das heißt: während in der europäischen Raumentwicklungspolitik das Prinzip einer evidence-based policy gepredigt wird, muss man in Österreich eigentlich von einer evidence-ignoring policy sprechen - was im Übrigen auch in anderen politischen Aufgabenbereichen zu beobachten ist.

Im Übrigen wird die dahinter liegende verwirrende Kompetenzlage nicht als Problem erkannt. Sie bildet vielmehr die willkommene Arena für die machtpolitischen Auseinandersetzungen zwischen verschiedenen Planungsträgern. Das kann sogar bis zu einer völligen Umkehrung der normalen hierarchischen Logik von Gesetz, Verordnung und Projektgenehmigung gehen. So wurde im Streitfall des Semmering-Basistunnels in Niederösterreich das (Landes-)Naturschutzgesetz mehrfach abgeändert, um damit die eisenbahnrechtliche Trassengenehmigung seitens des Bundes zu unterlaufen.

Auch im Bereich der Raumentwicklungspolitik zeigt sich damit der meines Erachtens generell zu beobachtende Trend zur Umkehrung des Ziel-Mittel-Verhältnisses in der Politik. Nicht die Ziele sind es, denen sich eine Politik mit ihrem Instrumentarium 
verpflichtet, sondern die Ziele werden disponibel und zum Instrument einer ausschließlich machtorientierten Politik. In dieser Situation läuft Raumplanung Gefahr, mit ihren Zukunftsbildern zur Auslagendekoration für ein politisches Geschäft zu verkommen, das im Hinterzimmer auf banale Weise die üblichen Einzelinteressen verfolgt.

\section{Zur Neuorientierung am Gemeinwohl}

Worum geht es eigentlich im Verhältnis Raumplanung - Gesellschaft - Politik, noch immer, jetzt nach mehr als 50 Jahren? Dazu ist zunächst auf den Begriff des Gemeinwohls zurückzugreifen, der ja in gesetzlichen Aufgabenbeschreibungen von Raumordnung enthalten ist. Er spielt meines Erachtens für die Raumplanung eine zentrale Rolle. Allerdings ist der Begriff aus dem allgemeinen gesellschaftspolitischen Diskurs völlig verschwunden.

Es fehlt offenbar überhaupt jegliche Vorstellung von Gemeinwohl in dieser aktuellen Gesellschaft; damit aber erst recht auch eine Vorstellung darüber, wie Gemeinwohl oder wie allgemein akzeptierte Grundwerte wie Solidarität und Gerechtigkeit im Raum umzusetzen wären. Das Schwierige dabei ist, dass sich diese Prinzipien erst im Anwendungsfall konkretisieren lassen.

Gerade die Raumordnung aber scheint dazu berufen, sich um deren Konkretisierung zu bemühen, weil erstens das Gemeinwohl eigentlich die wesentliche gesellschaftspolitische Legitimation der Raumplanung ist - Raumordnung ist die räumliche Gemeinwohlvorsorge schlechthin - und weil zweitens gerade an den Problemen im Raum relativ leicht verständlich gemacht werden kann, worum es beim Gemeinwohl geht.

Gemeinwohl ist ganz offensichtlich nicht die Summe aller Einzelinteressen. Im Falle einer wünschenswerten Siedlungsstruktur heißt das zum Beispiel: Die endlose Addition des freistehenden Einfamilienhauses - offensichtlich das Symbol der „freien Entfaltung der Persönlichkeit ... in der Gemeinschaft" (Salzburger Raumordnungsgesetz) - kann sehr wohl zum Problem werden. Ebenso verhält es sich im Falle des Individualverkehrs oder auch des Tourismus, um nur zwei weitere Beispiele zu nennen.

Mit jedem Grundstück, mit jedem Standort ist also nicht nur ein individuelles Interesse verbunden, sondern grundsätzlich auch ein Gemeinwohlinteresse, gleichsam als Folie darüber. Ich erinnere an das deutsche Grundgesetz, in dem es heißt: „Eigentum verpflichtet. Sein Gebrauch soll auch dem Allgemeinwohl dienen.“ Welches Gewicht dieses hat, und ob es zum individuellen Interesse im Konflikt steht, ist nur am Einzelfall bestimmbar. Die Bandbreite reicht von null Einschränkung bis zum Extremfall einer sehr weitgehenden Einschränkung wie im Fall des Naturschutzes. Wenn Gemeinwohl also nur am Einzelfall definierbar ist, dann muss es dort aber auch in die Erwägung tatsächlich eingebracht werden, ausdrücklich und argumentiert.

Die Interpretation des Gemeinwohls wird auch dadurch noch einmal komplexer, dass es jeweils auf den verschiedenen Ebenen zu konkretisieren ist. Es ergibt sich 
ja auch aus der Summe der Gemeindeinteressen nicht das Landesinteresse - und so verhält es sich auch auf der Bundesebene. Für die Abstimmung zwischen den Ebenen gilt, dass es keine technische Lösung gibt, sondern nur die politische Verhandlung. Das wäre im Übrigen ein Thema für sich.

\section{Zu eventuell geeigneten Zukunftsstrategien}

Raumplanung ist ein politisches Geschäft. Die Raumplanungsbehörde ist nicht unabhängig wie ein Gericht, sondern steht unter politischer Verantwortung. Damit ist sie auch dem politischen Kalkül unterworfen. Und dieses orientiert sich an den (tatsächlichen oder vermeintlichen) Wertorientierungen der jeweiligen politischen Klientel. Das gilt für alle Ebenen.

Änderungen an der beschriebenen planungsfeindlichen Einstellung des politischen Personals werden meines Erachtens nur über den „Umweg“ öffentlichen Drucks zu erreichen sein. Wenn es also darum geht, in der Öffentlichkeit eine größere Akzeptanz für Raumplanung zu erzielen, so empfiehlt sich eine strategische Umorientierung. Raumplanung müsste sich endlich vom nicht einlösbaren Anspruch der Verantwortung für die (optimale) Gesamtgestaltung des Raumes befreien und sich stärker als Mittel zur selektiven, aber gezielten Durchsetzung mehrheitsfähiger allgemeiner Grundsätze verstehen und darstellen. Dazu gehören

- Einsparen öffentlicher Mittel;

- Vermeiden von Ressourcenvergeudung;

- bestmögliche Nutzung vorhandener Potenziale;

- Reduktion von Umweltbelastungen

in den verschiedenen Politikbereichen.

Die Frage ist doch heute, woraus - im Wettbewerb mit anderen politischen Aufgabenfeldern (wie vor allem der Umweltpolitik) - Raumplanung eigentlich ihre spezifische politische Legitimation bezieht. Die Zielbestimmungen der Landes-Raumordnungsgesetze reichen dazu ganz offensichtlich nicht aus (sie werden ja auch nicht ernsthaft verfolgt). Nutzen oder Mehrwert der Raumplanung müssen den je relevanten Partnern vermittelt werden. Sie liegen:

- im Messen der Auswirkungen raumwirksamer Maßnahmen in „,räumlicher Währung“ (betroffene Standorte, Verbindungen, Gebiete);

- im Erfassen externer Effekte der jeweiligen ,eigenen“ und „fremden“ Maßnahmen (,Verträglichkeitsprüfungen“ verschiedener Art sind ein aktuelles Marktsegment der Planung);

- darin, dass Raumplanung die Voraussetzung für das Konfliktmanagement von raumwirksamen Ansprüchen und Maßnahmen schafft;

- und nicht zuletzt im Aufzeigen von latenten Synergiepotenzialen unkoordinierter Maßnahmenfelder. 
Für die Raumplanung muss also die zentrale Aufgabe das Gewinnen von Mehrheiten auf dem Meinungsmarkt sein. Als wichtigstes Produkt der Planung ist demnach immer weniger der Entwurf von Anordnungen zu sehen, sondern die zweckorientierte Information zur Überzeugung der Adressaten. Pläne haben dann weniger die Rolle von Handlungsanleitungen, sondern die von „Stimmungsmachern“, Diskursstartern oder auch nur von Instrumenten der Öffentlichkeitsarbeit der Planungsträger. Dafür sind in aller Regel Analysen, Prognosen, Szenarien und Leitbilder zunächst wichtiger als behördliche Pläne.

Gerade im Rahmen eines Geographentags erscheint es angebracht zu betonen, dass die Grundvoraussetzung für die Mehrheitsfähigkeit eine Raumwahrnehmung ist, die - auf der jeweiligen Ebene - die lebensweltlichen Zusammenhänge im Raum erkennt und dementsprechend den Raum als Verantwortungsdimension in den politischen Diskurs einführt; durchaus in Gegenüberstellung zu den üblicherweise dominierenden sektoralen Zuständigkeiten.

Am Schluss soll auf ein diesbezüglich interessantes Beispiel hingewiesen werden.

\section{Konkrete Erfahrung: Betroffene als Geographen}

In einem eindrucksvollen Planungsprozessprojekt, das zwischen 2004 und 2006 29 Gemeinden im Vorarlberger Rheintal mit insgesamt ca. 235.000 Einwohnern erfasste, ging es darum, überhaupt erst das Verständnis und die Bereitschaft für Regionalplanung zu erreichen (vgl. Abb. 1, 2). In diesem Tal mit der nach Wien höchsten Bevölkerungsdichte in Österreich sind die Gemeindegrenzen mitten im geschlossenen Siedlungsgefüge vielfach nur an den Ortstafeln erkennbar. Die Mehrzahl aller Bewohner verlässt für zahlreiche Funktionen täglich die eigene Gemeinde. Es werden also höchst intensive regionale Verflechtungen gelebt, trotzdem war die Region als gemeinsamer Lebensraum zuvor nicht wahrgenommen worden. In den Köpfen war nur die eigene - noch dazu ländlich verstandene - Gemeinde. In einem breiten Beteiligungsprozess mit insgesamt 800 Beteiligten konnte eine neue Wahrnehmung der Lebensrealität erreicht werden, mit detaillierten Karten, beispielsweise über die arbeitsbezogenen Verflechtungen oder über die freizeitbezogenen Pfade der Jugendlichen. Ein wesentliches Mittel waren dafür die mentalen Landkarten, die von den Workshop-Teilnehmern selbst zu verschiedenen Lebensbezügen hergestellt wurden. Die neue Sichtweise kommt etwa in folgenden Worten einer Teilnehmerin zum Ausdruck: „Im Grunde genommen ist das Rheintal schon eine Region - zumindest im Alltag. Ohne dass man es merkt, lebt man mindestens in fünf bis sechs Gemeinden.“

Seit zwei Jahren wird als Konsequenz aus dem Projekt der „Regionale Kontrakt Rheintal“, abgeschlossen zwischen Landesregierung und Gemeinden, umgesetzt. Derzeit aktuelle Maßnahmen sind die Themen Kinderbetreuung und Betreuung älterer Menschen in Gemeindekooperation sowie die Siedlungsverdichtung an Bahnhofsstandorten und die Vorbereitung von regionalen Betriebsgebieten. 


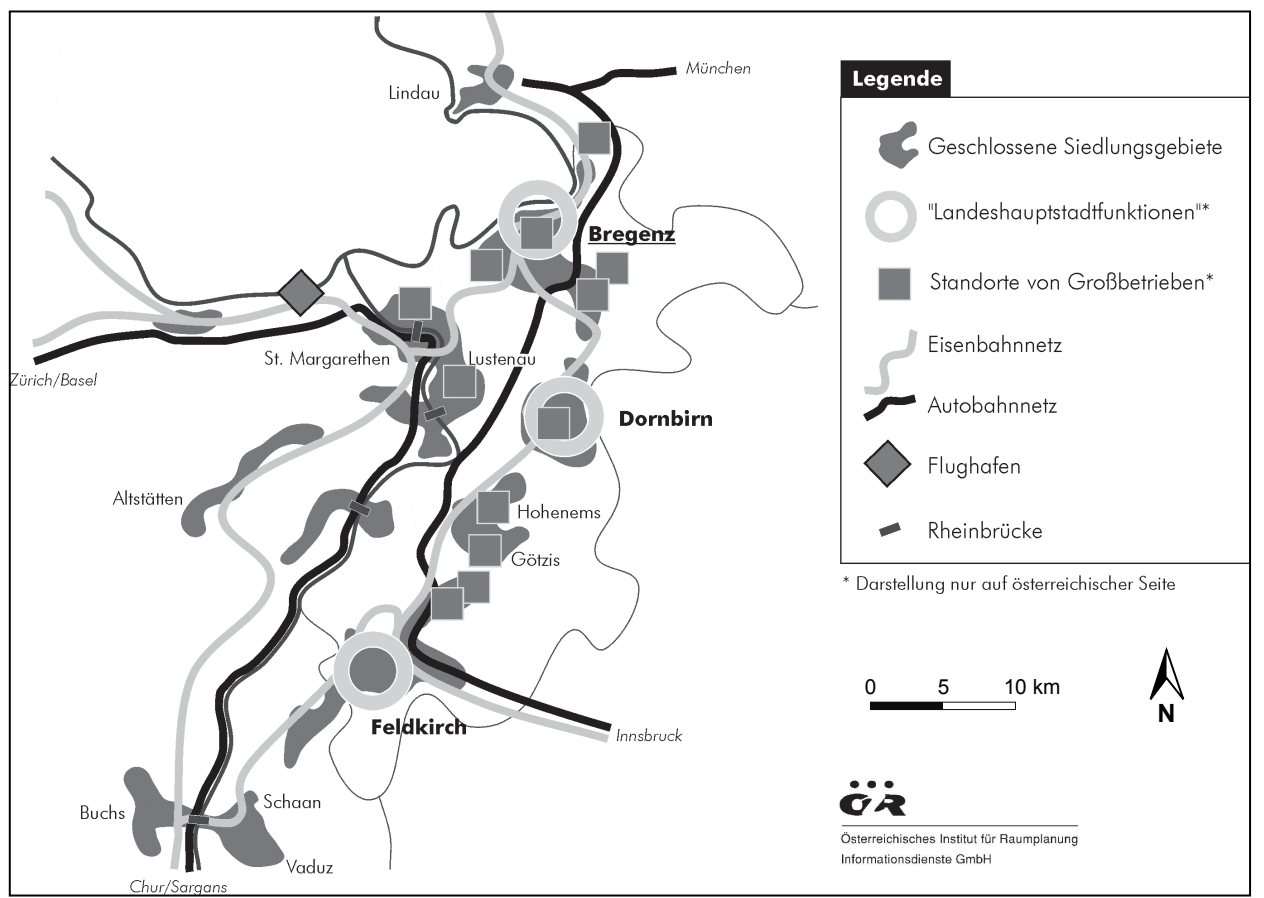

Abb. 1: Schauplatz des Planungsprozessprojekts Vorarlberger Rheintal

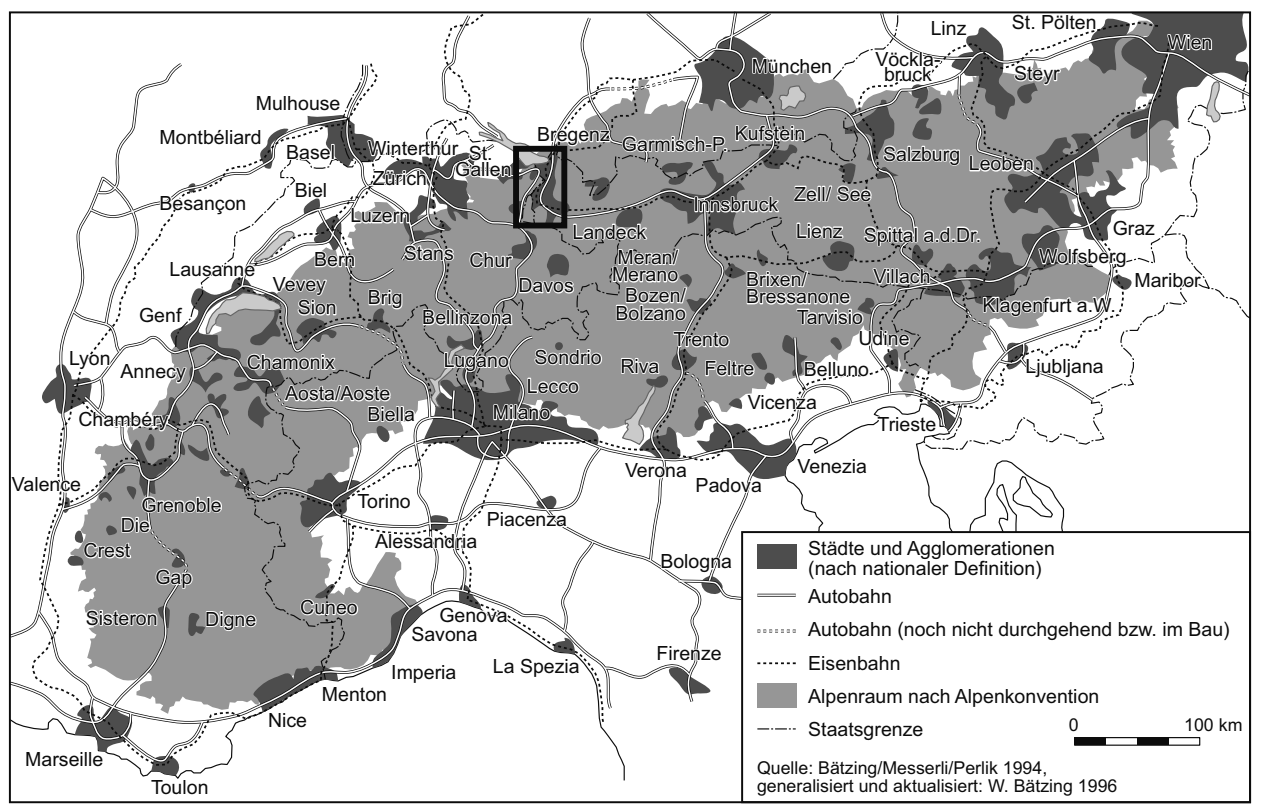

Abb. 2: Das Vorarlberger Rheintal im Alpenraum 
Die im Rahmen des Projekts „vision rheintal“ praktizierte Vorgangsweise kann unter anderem durchaus als angewandte Geographie pur gesehen werden, und zwar in Anwendung durch die sogenannten Betroffenen selbst.

An diesem Beispiel wird Planung als Instrument einer lernenden Gesellschaft erkennbar. Ein dieser Vision verpflichtetes Planungssystem beruht wesentlich auf der Wahrnehmung der Tatsachen, auf der Überzeugung der Akteure sowie auf der gezielten Auswertung der Erfahrungen der Anwendung der Planungsinstrumente.

Als zusammenfassende Schlussbemerkung sei noch einmal hervorgehoben: In Österreich haben wir es nicht mit einer erst kürzlich aufgetretenen, sondern mit einer gleichsam systemimmanenten Krise der Raumplanung zu tun. Um sie zu überwinden, wäre eine strategische Umorientierung notwendig, die weg führt von der Behördenplanung und hin lenkt zur politischen Meinungsbildung im öffentlichen Raum, in Richtung einer von Mehrheiten getragenen räumlichen Gemeinwohlvorsorge.

\section{Literaturverzeichnis}

Faludi A., W aterhout B. (2006), Introducing Evidence-Based Planning. In: disP - The Planning Review, 2, S. 4-13. Zürich, ETH Zürich.

LAND Vorarlberg und 29 namentlich angeführte Rheintalgemeinden (Hrsg.) (2006), vision rheintal Dokumentation 2006, Räumliche Entwicklung und regionale Kooperation im Vorarlberger Rheintal, Ergebnisse des Leitbildprozesses. Eigenverlag der Raumplanungsabteilung beim Amt der Vorarlberger Landesregierung. - http://www.vision-rheintal.at

Schindegger F. (1999), Raum. Planung. Politik. Ein Handbuch zur Raumplanung in Österreich. Wien, Böhlau.

SchindegGer F. (2003), Sachwalter der räumlichen Verantwortlichkeit. In: Raum. Österr. Zeitschrift f. Raumplanung u. Regionalpolitik, 52, S. 20-24.

Schindegger F. (2006), Raumplanung - Gesellschaft - Politik: ein Verhältnis nach 50 Jahren. In: Raum. Österr. Zeitschrift f. Raumplanung u. Regionalpolitik, 64, S. 44-49.

SchindegGer F. (2009), Raumordnung im Regierungsprogramm. In: konstruktiv, Zeitschrift d. Bundeskammer d. Architekten u. Ingenieurkonsulenten, 272, März/April, S. 30-31.

Schönwandt W. (2002), Planung in der Krise? Theoretische Orientierungen für Architektur, Stadt- und Raumplanung. Stuttgart, Kohlhammer.

Wichmann T. (2008), Planung und Adaption. Strategieentwicklung in Regionen, Organisationen und Netzwerken. Dortmund, Rohn. 